\title{
Transpancreatic biliary sphincterotomy versus double guidewire in difficult biliary cannulation: a randomized controlled trial
}

Authors

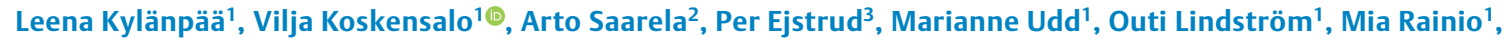
Andrea Tenca ${ }^{4}$, Jorma Halttunen ${ }^{1}$, Gunnar Qvigstad ${ }^{5}$, Urban Arnelo ${ }^{6}$, Niklas Fagerström ${ }^{6}$, Truls Hauge ${ }^{7}$, Lars Aabakken $^{8}$, Juha Grönroos ${ }^{9}$

Institutions

1 Abdominal Center, Gastroenterological Surgery, Helsinki University Hospital and University of Helsinki, Helsinki, Finland

2 Department of Gastrointestinal Surgery, Oulu University Hospital, Oulu, Finland

3 Department of Gastrointestinal Surgery, Aalborg University Hospital, Aalborg, Denmark

4 Abdominal Center, Gastroenterology, Helsinki University Hospital and University of Helsinki, Helsinki, Finland

5 Department of Gastroenterology and Hepatology, St. Olavs Hospital, Trondheim University Hospital, Trondheim, Norway

6 Department of Upper Gastrointestinal Diseases, Karolinska University Hospital, Stockholm, Sweden

7 Department of Gastroenterology, Oslo University Hospital, Oslo, Norway

8 Department of Medical Gastroenterology, Rikshospitalet University Hospital, Oslo, Norway

9 Division of Digestive Surgery and Urology, Turku University Hospital and University of Turku, Turku, Finland

submitted 17.5.2020

accepted after revision 27.10.2020

published online 13.1.2021

\section{Bibliography}

Endoscopy 2021; 53: 1011-1019

DOI 10.1055/a-1327-2025

ISSN 0013-726X

(c) 2021. Thieme. All rights reserved.

Georg Thieme Verlag KG, Rüdigerstraße 14,

70469 Stuttgart, Germany

Table 1s, Fig. 1s-3s

Supplementary material is available under https://doi.org/10.1055/a-1327-2025
丹 Scan this QR-Code for the author commentary.

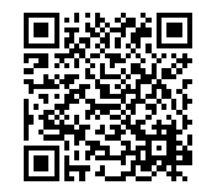

Corresponding author

Vilja Koskensalo, MD, Haartmaninkatu 4, Helsinki PL 340, 00029 HUS, Finland

vilja.koskensalo@helsinki.fi

\section{ABSTRACT}

Background Difficult biliary cannulation in endoscopic retrograde cholangiopancreatography (ERCP) increases the risk of post-ERCP pancreatitis (PEP). The purpose of this prospective, randomized, multicenter study was to compare two advanced rescue methods, transpancreatic biliary sphincterotomy (TPBS) and a double-guidewire (DGW) technique, in difficult common bile duct (CBD) cannulation. Methods Patients with native papilla and planned CBD cannulation were recruited at eight Scandinavian hospitals. An experienced endoscopist attempted CBD cannulation with wire-guided cannulation. If the procedure fulfilled the definition of difficult cannulation and a guidewire entered the pancreatic duct, randomization to either TPBS or to DGW was performed. If the randomized method failed, any method available was performed. The primary end point was the frequency of PEP and the secondary end points included successful cannulation with the randomized method.

Results In total, 1190 patients were recruited and 203 (17.1\%) were randomized according to the study protocol (TPBS 104 and DGW 99). PEP developed in 14/104 patients $(13.5 \%)$ in the TPBS group and $16 / 99$ patients (16.2\%) in the DGW group $(P=0.69)$. No difference existed in PEP severity between the groups. The rate of successful deep biliary cannulation was significantly higher with TPBS (84.6\% [88/104]) than with DGW (69.7\% [69/99]; $P=0.01)$.

Conclusions In difficult biliary cannulation, there was no difference in PEP rate between TPBS and DGW techniques. TPBS is a good alternative in cases of difficult cannulation when the guidewire is in the pancreatic duct. 


\section{Introduction}

In endoscopic retrograde cholangiopancreatography (ERCP), difficult common bile duct (CBD) cannulation is defined according to the European Society of Gastrointestinal Endoscopy (ESGE) criteria as more than 5 minutes attempting to cannulate, more than five contacts with the papilla, or more than one unintended entry into the pancreatic duct (PD) or opacification in wire-guided cannulation (5-5-2 definition) [1]. After persistent attempts with standard methods (e.g. wire-guided cannulation) have failed, access to the CBD often requires the application of advanced cannulation techniques, such as sphincterotome precut, needle-knife precut, and pancreatic guidewire-assisted cannulation with a single wire, proceeding to transpancreatic biliary sphincterotomy (TPBS) or a doubleguidewire technique (DGW), pancreatic stenting or papillectomy [1]. However, difficult cannulation and repeated attempts increase the risk of post-ERCP pancreatitis (PEP) to $10.8 \%-16.2 \%[1-4]$.

The aim of the present study was to compare two different advanced cannulation techniques, TPBS and DGW, in terms of PEP and successful cannulation.

\section{Methods}

\section{Study design}

The recruited patients underwent ERCP between 2015 and 2019 at eight hospitals: Helsinki University Hospital, Oulu University Hospital, Aalborg University Hospital, St. Olavs Hospital in Trondheim University Hospital, Karolinska University Hospital, Oslo University Hospital, Oslo's Rikshospitalet University Hospital, and Turku University Hospital.

The ethical committees of each hospital approved the study protocol. The study protocol conformed to the ethical guidelines of the 2008 Declaration of Helsinki. The original study protocol is presented in Fig. $\mathbf{1} \mathbf{s}$ in the online-only supplementary material.

\section{Patients}

The inclusion criteria for recruitment were native papilla, ERCP intended for CBD cannulation, and age over 18 years. The exclusion criteria were acute pancreatitis and no consent for participation in the study. The inclusion criteria for randomization were difficult biliary cannulation and the guidewire entering the PD.

\section{Interventions}

ERCPs were performed by experienced endoscopists. PEP prophylaxis prior to ERCP (rectal nonsteroidal anti-inflammatory drugs [NSAIDs]-100 mg diclofenac or indomethacin for patients without contraindication) was recommended by the study protocol, but implementation was left to the preference of the endoscopist. In addition, prophylactic pancreatic stenting was performed at the discretion of the endoscopist.

Patients fulfilling the inclusion criteria were included. Written informed consent was obtained from all patients prior to ERCP.

\section{Randomization}

A randomization list was produced with a random-number generator by using random blocks (size 20) with 1:1 allocation. The allocation of patients was concealed using sequentially numbered, opaque, sealed envelopes. A nurse opened the envelope only after the procedure fulfilled the difficult cannulation criteria and the wire had entered the PD.

\section{ERCP procedure}

The TPBS technique was adopted in participating hospitals under the guidance of an expert TPBS endoscopist (J.H.) The cannulation study protocol (Fig.1s) was presented at biannual Scandinavian Association for Digestive Endoscopy (SADE) research meetings (March and September 2015-2019) in order to inform and encourage more endoscopists and centers to participate in the study, and continuous education assured the quality of TPBS and DGW techniques performed by experienced endoscopists.

Only wire-guided cannulation was used for initial cannulation attempts. The primary cannulation was defined as successful deep biliary cannulation when the guidewire was inside the CBD. If the primary cannulation failed and the 5-5-2 definition of difficult cannulation was fulfilled, the preceding factor leading to difficult cannulation was recorded. All procedures meeting the difficult cannulation criteria and involving the guidewire entering the PD were randomized to either TPBS or DGW.

The randomized method was performed without removing the guidewire from the PD. A time limit of 15 minutes was allowed before the method was regarded as unsuccessful. After 15 minutes, an endoscopist could either change the method or continue the cannulation attempts with the first randomized method.

The success or failure of the randomized method, any additional rescue methods, and the time for successful cannulation or total time used (in cases of final failure) were recorded.

\section{The TPBS technique}

The aim of TPBS is to incise the septum between the PD and the CBD in order to expose the bile duct orifice. If a guidewire unintentionally enters the PD, TPBS is performed with a regular sphincterotome and a pure-cut electrosurgical current, toward the 11 or 12 o'clock position. The cut either opens the CBD or reveals the anatomy of the ampulla ( $\triangleright$ Fig. 1 and $\triangleright$ Fig.2a, green cut indicates TPBS technique). A further oblique cut toward the 10 o'clock position, using a needle-knife and starting from the upper end of the previous TPBS, can be performed after the TPBS technique, if required ( $\mathbf{F i g} \mathbf{2}$, red cut indicates needle-knife technique). The intention is to cut across to the CBD and expose the lumen [5].

\section{The DGW technique}

The DGW technique involves deep PD cannulation using the first guidewire, which may straighten the CBD, thus allowing CBD cannulation with the second guidewire. Therefore, the original guidewire that entered the PD remains in place in order to 


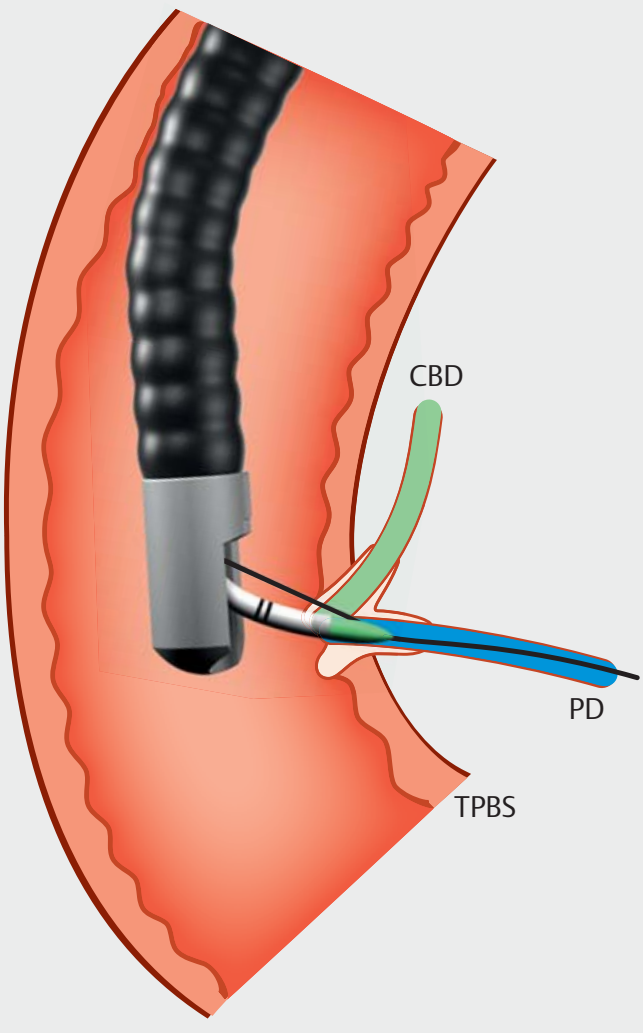

- Fig. 1 Transpancreatic biliary sphincterotomy technique. CBD, common bile duct; PD, pancreatic duct.

aid orientation and further attempts to cannulate the CBD are performed using wire-guided cannulation with the second guidewire (> Fig. 3 ).

\section{Post procedure}

All patients remained in the hospital for at least 4 hours after ERCP and were monitored for the development of PEP, bleeding, cholangitis, perforation, or any other complication.

Serum or plasma amylase was measured 4 hours after the procedure. Thereafter, the patient was discharged at the discretion of the physician. If the patient remained in the hospital overnight, serum or plasma amylase was assayed the following morning.

\section{Definitions and outcomes}

The primary outcome was the rate of PEP. Secondary outcomes were successful cannulation with the randomized method within 15 minutes and in total. All other results were considered descriptive.

PEP was defined as the presence of abdominal pain attributable to acute pancreatitis, together with a need for unplanned hospitalization or an extension of a planned hospitalization by at least 2 days, and serum or plasma amylase at least three times above the upper limit of normal (ULN) at 24 hours after the procedure [6]. PEP was classified as mild, moderate, or se-

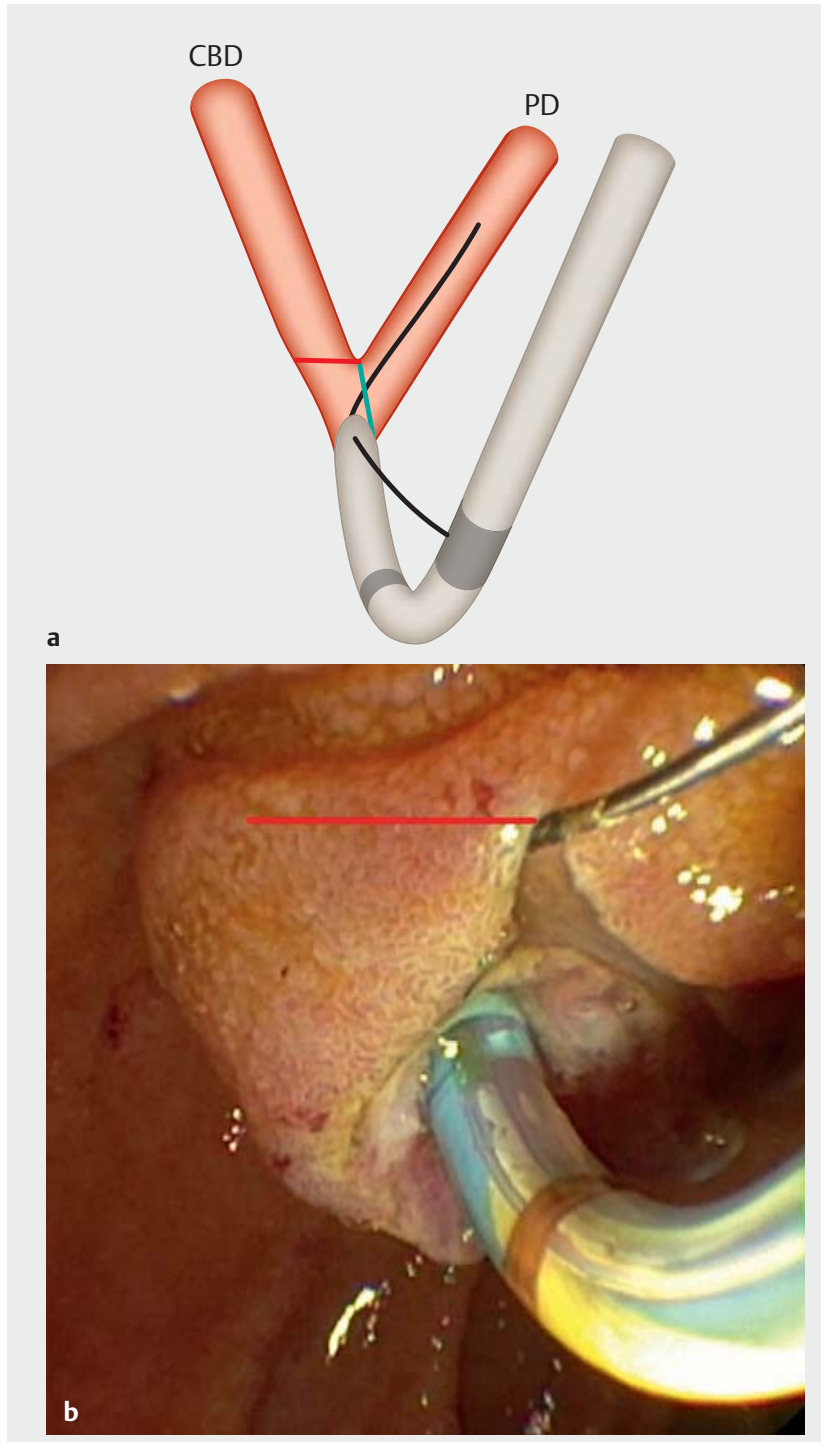

- Fig. 2 Transpancreatic biliary sphincterotomy (TPBS) and additional needle-knife technique. a Schematic. b Endoscopic view. After TPBS, access into the common bile duct (CBD) may succeed either through the papilla, through the upper corner of the cut, or after oblique needle-knife cut across the CBD. PD, pancreatic duct; green line, line of sphincterotomy; red line, additional needle-knife cut.

vere if the patient had to stay in hospital for less than 4 days, 4 10 days, or more than 10 days, respectively [6].

Success with the randomized method was defined as success when the guidewire entered the CBD. Failure was defined as a failure with the cannulation method used. Final failure was defined as no access to the CBD with any of the methods used.

Any complications, admission times, and any additional care during the first 30 days after the procedure were recorded. In addition, patients were advised to contact the physician at any time if they noticed symptoms such as fever or abdominal pain. We assumed that absence of documented adverse events in the patient records meant that no adverse events occurred. 

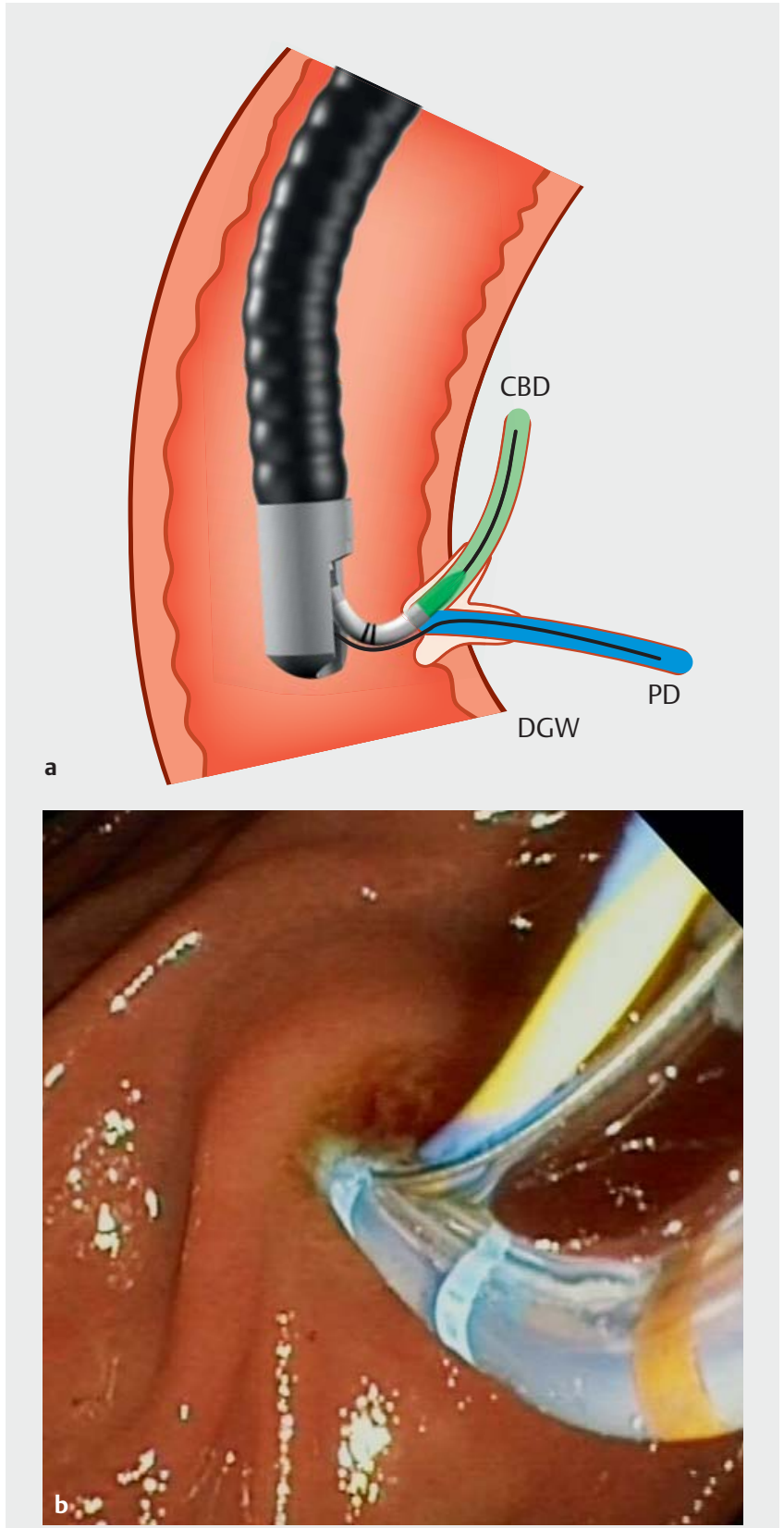

- Fig. 3 Double-guidewire technique. a Schematic. b Endoscopic view. CBD, common bile duct; PD, pancreatic duct.

\section{Sample size calculation}

The sample size calculation assumed that $70 \%$ of primary cannulations would be successful within the limits of difficult cannulation (i.e. not described as difficult), $30 \%$ of all cannulations would be difficult and, of these two-thirds would be randomized (i. e. $20 \%$ of total). According to previous studies, the PEP rate was lower following TPBS $(0 \%-10.8 \%[5,7,8])$ than after DGW $(17 \%-38.2 \%[8-11])$. If the $0 \%$ rates are ignored, the mean rates were $10 \%$ and $25 \%$ for TPBS and DGW, respectively. The sample size calculation was performed with power $=0.8$ and alpha $=0.05$, resulting in 97 patients per randomized group [12].

\section{Statistical analysis}

Data were analyzed on an intention-to-treat basis and randomized patients were analyzed according to their original groups. Analyses were performed using IBM SPSS Statistics for Macintosh, v25.0 (IBM Corp., Armonk, New York, USA) and with R v4.00 software (R Foundation for Statistical Computing, Vienna, Austria).

The primary end point variable was compared using chisquared and Fisher's exact tests. Statistical significance was assayed as a two-tailed $P$ value of $<0.05$. All other statistical tests of outcome results were considered as secondary and their results were taken as descriptive only. In descriptive statistics, continuous variables were described as median (range) or as interquartile range (IQR), and categorical variables were described as number of cases and proportion. Descriptive continuous and ordinal variables were compared using the MannWhitney $U$ test and categorical variables were compared using Fisher's exact test.

\section{Results}

\section{Patients}

A total of 1190 patients underwent ERCP and were recruited between September 2015 and April 2019. The PEP rate among nonrandomized patients who had data available was $4.7 \%$ (42) $890)$. The deep biliary cannulation success rate of all ERCPs was $98.8 \%(1176 / 1190)$.

\section{Difficult cannulation}

In total, 203 patients (17.1\%) met the criteria of difficult cannulation and were randomized (104 to TPBS and 99 to DGW) ( Fig.4) The number of recruited patients in each hospital is shown in Table1s. The demographic characteristics of the randomized patients and ERCP procedures are presented in - Table 1. The median duration of cannulation was 12.5 minutes (IQR 12.7) in the TPBS group and 14.1 minutes (IQR 16.5) in the DGW group $(P=0.45)$.

The cannulation events preceding randomization were (TPBS vs DGW): PD passage/opacification in 82 (78.8\%) vs. 68 $(68.7 \%)$ patients $(P=0.12)$; more than 5 minutes spent attempting to cannulate the CBD in $48(46.2 \%)$ vs. 52 (52.5\%) patients $(P=0.40)$; and more than five contacts with the papilla in $35(33.7 \%)$ vs. $30(30.3 \%)$ patients $(P=0.65)$, respectively.

A diverticulum was present in 17/203 (8.4\%) patients (7 patients [6.7\%] in the TPBS group and 10 patients [10.1\%] in the DGW group). In most cases, the papilla was situated at the edge of the diverticulum (11/17 [64.7\%]). When a diverticulum was present, cannulation succeeded with the allocated randomized method in $6 / 7$ patients $(85.7 \%)$ in the TPBS group and in 5/10 patients $(50.0 \%)$ in the DGW group $(P=0.30)$. All cannulations were finally successful in the TPBS group whereas one failed in the DGW group $(P>0.99)$.

Pancreatic or biliary malignancy was the indication for ERCP in $39 / 104$ patients (37.5\%) in the TPBS group and 43/99 patients (43.4\%) in the DGW group. In these patients, cannulation succeeded with the allocated randomized method in 32/39 


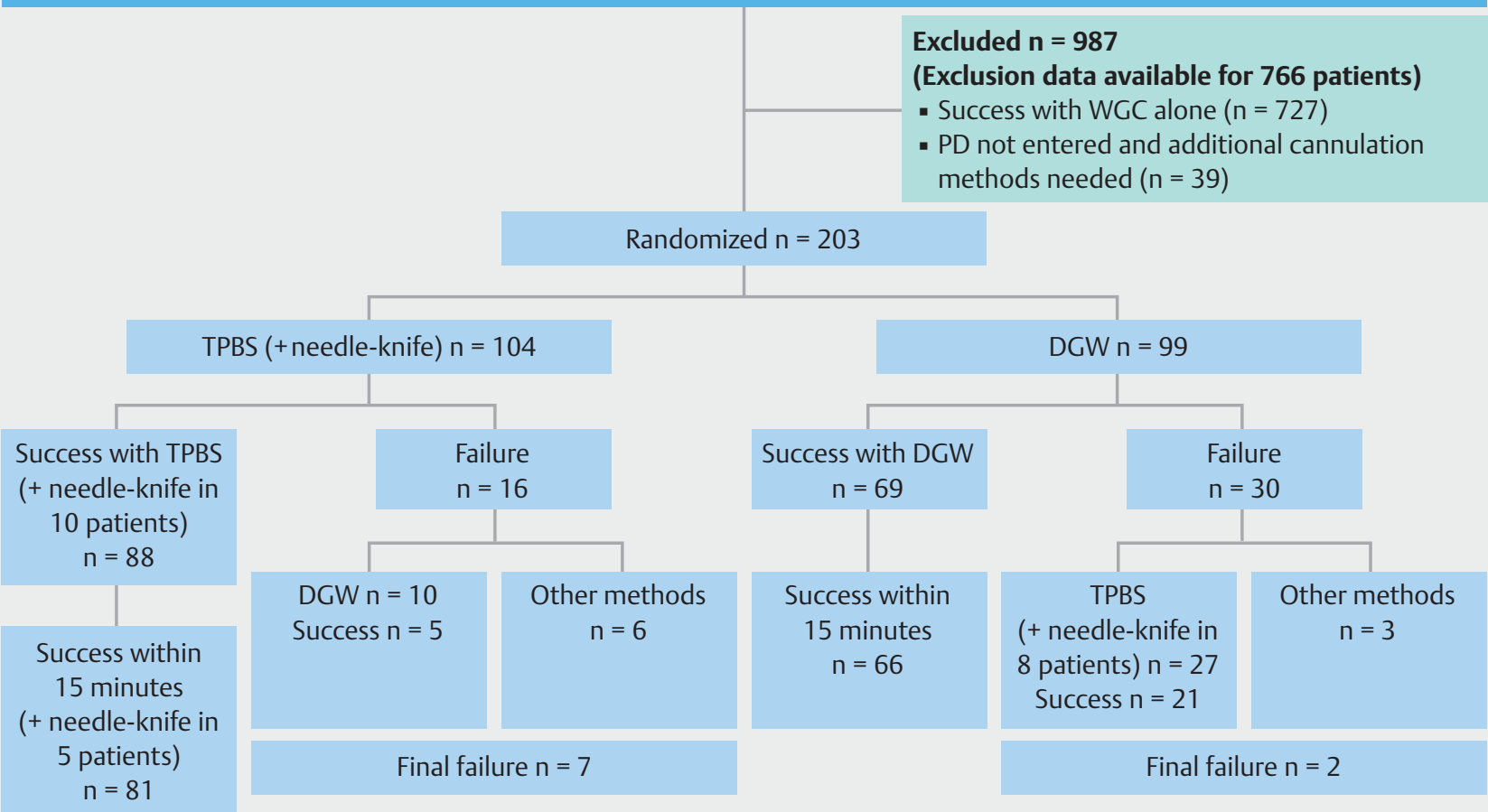

Fig. 4 CONSORT flow diagram of recruited and randomized patients. Data are presented as the number of patients. ERCP, endoscopic retrograde cholangiopancreatography; WGC, wire-guided cannulation; PD, pancreatic duct; TPBS, transpancreatic biliary sphincterotomy; DGW, double-guidewire technique.

- Table 1 Demographic data of the randomized patients and endoscopic retrograde cholangiopancreatography procedures.

\begin{tabular}{|c|c|c|c|}
\hline & TPBS $n=104$ & DGW n= 99 & $P$ value \\
\hline Age, median (range), years & $66(21-97)$ & $68(24-92)$ & 0.47 \\
\hline Female sex, n (\%) & $46(44.2)$ & $59(59.6)$ & 0.04 \\
\hline NSAID as a PEP prophylaxis, n (\%) & $82(78.8)$ & $82(82.8)$ & 0.72 \\
\hline \multicolumn{4}{|l|}{ Indication for ERCP, n (\%) } \\
\hline - CBD stones & $47(45.2)$ & $40(40.4)$ & 0.48 \\
\hline - Stricture & $43(41.3)$ & $48(48.5)$ & 0.33 \\
\hline - PSC & $6(5.8)$ & $6(6.1)$ & $>0.99$ \\
\hline - Post cholecystectomy (bile leakage) & $8(7.7)$ & $9(9.1)$ & 0.80 \\
\hline - Post LTX (stricture) & $0(0)$ & $1(1.0)$ & 0.49 \\
\hline - Post other surgery & $0(0)$ & $2(2.0)$ & 0.24 \\
\hline - Other & $8(7.7)$ & $9(9.1)$ & 0.80 \\
\hline Prophylactic pancreatic stent & $9(8.7)$ & $11(11.1)$ & 0.64 \\
\hline
\end{tabular}

TPBS, transpancreatic biliary sphincterotomy; DGW, double-guidewire technique; NSAID, nonsteroidal anti-inflammatory drug; PEP, post-ERCP pancreatitis; ERCP, endoscopic retrograde cholangiopancreatography; CBD, common bile duct; PSC, primary sclerosing cholangitis; LTX, liver transplantation. 


\begin{tabular}{|c|c|c|c|}
\hline & TPBS $n=104$ & DGW n= 99 & $P$ value \\
\hline PEP, n (\%) & $14(13.5)$ & $16(16.2)$ & 0.69 \\
\hline PEP severity, $n$ (\%) & & & 0.19 \\
\hline - Mild & $7(6.7)$ & $9(9.1)$ & $>0.99$ \\
\hline - Moderate & $4(3.8)$ & $7(7.1)$ & 0.47 \\
\hline - Severe & $3(2.9)$ & $0(0.0)$ & 0.09 \\
\hline Other complications total, $\mathrm{n}(\%)^{*}$ & $3(2.9)$ & $5(5.1)$ & 0.49 \\
\hline - Bleeding & $0(0.0)$ & $1(1.0)$ & 0.49 \\
\hline - Perforation & $2(1.9)$ & $1(1.0)$ & $>0.99$ \\
\hline - Cholangitis & $1(1.0)$ & $2(2.0)$ & 0.61 \\
\hline - Other infection & 0 & $2(2.0)$ & 0.24 \\
\hline
\end{tabular}

(82.1\%) in the TPBS group and in 29/43 (67.4\%) in the DGW group. The final cannulation failure rate was not statistically different between the two methods in this patient group (3/39 [7.7\%] with TPBS vs. 0/43 [0.0\%] with DGW; $P=0.10)$.

In 4/104 patients (3.8\%) in the TPBS group and 2/99 patients $(2.0 \%)$ in the DGW group, trainees were involved in cannulation prior to randomization. In all these cases, trainees were supervised by experts.

\section{Primary outcome - rate of PEP}

ERCP complications with TPBS and DGW are presented in - Table 2 . The PEP rate was $14.8 \%$ in the whole cohort of patients with difficult cannulation $(n=203)$. There was no significant difference between the TPBS and DGW techniques in terms of PEP rate (13.5\% vs. $16.2 \%$, respectively) or the severity of PEP. Among patients with PEP, the median cannulation time was 10.6 minutes (range $6.0-33.9$ ) in the TPBS group and 10.9 minutes (range $3.1-77.7)$ in the DGW group $(P=0.61)$.

PEP developed in $25 / 164$ patients $(15.2 \%)$ in the NSAID group and in $5 / 37$ patients $(13.5 \%)$ in the non-NSAID group ( $P$ $>0.99)$.

\section{Cannulation success}

Within 15 minutes, deep biliary cannulation was achieved in $81 / 104$ patients $(77.9 \%$ ) in the TPBS group and in 66/99 patients $(66.7 \%)$ in the DGW group $(P=0.09)$ ( $\triangleright$ Fig. 4 ). If the time limit is disregarded, the success rate of deep biliary cannulation was significantly higher with TPBS $(84.6 \%, n=88)$ than with DGW (69.7\%, $\mathrm{n}=69 ; P=0.01)$. In successfully cannulated TPBS patients $(n=88)$, a needle-knife was also used in 10 procedures (11.4\%).

Median time to successful cannulation with the randomized method was 10.6 minutes (IQR 9.6) with TPBS and 9.3 minutes (IQR 9.9) with DGW $(P=0.52)$.

Cannulation with the randomized method failed in 16 patients in the TPBS group and in 30 patients in the DGW group
$(P=0.01)$. A needle-knife was also used with TPBS in 4/16 patients. Six TPBS patients and 16 DGW patients underwent a second rescue method after failure of the randomized method before the end of the study protocol time limit of 15 minutes. The additional rescue methods used in TPBS (13 patients) and DGW (30 patients) groups are presented in Fig. 2 s and Fig. $\mathbf{3 s ,}$ respectively. In the randomized DGW group, TPBS was used as a rescue method after failure in $27 / 30$ patients $(90.0 \%)$ and successful CBD cannulation was achieved in 21/27 (77.8\%). In the randomized TPBS group, DGW was used as a secondary rescue method in $10 / 16$ patients and as a tertiary method in one patient, with final success in 5/11 (45.5\%). When comparing the success with these rescue methods (TPBS $n=27$, DGW $n=$ 11 , none/other $n=8)$, TPBS seemed to succeed most often ( $P$ $=0.10)$. Final failure occurred in seven patients $(6.7 \%)$ in the TPBS group and two patients $(2.0 \%)$ in the DGW group $(P=$ $0.17)$.

\section{Discussion}

Using the ESGE 5 - 5 -2 definition of difficult cannulation [1], our study showed no difference in PEP rate between the two rescue cannulation methods of TPBS and DGW. However, successful CBD cannulation was significantly greater with the TPBS technique than with the DGW technique.

Primary cannulation success ranged from $70.6 \%$ to $88.2 \%$ in the participating hospitals. This variation might be due to differences in patient selection and/or the higher volume of more difficult procedures in some of the participating hospitals. In the present study, the rate of difficult cannulation was 203/1190 (17.1\%). In previous studies, however, difficult cannulation has varied between $1.4 \%$ and $49.5 \%$ [13-17]. In the present study, overall cannulation success was high at $95.6 \%$ in patients with difficult cannulation and $98.8 \%$ among all patients. 
PEP is the most common complication of ERCP. Mechanical trauma to the papilla Vateri and the pancreatic sphincter due to repeated cannulation attempts, hydrostatic injury by pancreatic fluids and contrast media, and thermal injury are probable cofactors in the development of PEP [18]. In unselected patients, the incidence of PEP is reported to be $3.5 \%-4.2 \%$ $[19,20]$. Difficult cannulation and repeated attempts increase this risk of PEP to $10.8 \%-16.2 \%$ [1-4]. In the present study, the PEP rate was $14.8 \%$ in difficult cannulation and $4.7 \%$ in all patients. The PEP rate was not significantly different between TPBS and DGW.

There are some commonly accepted patient- and procedure-related risk factors for PEP. Procedure-related factors include difficult cannulation [2, 21, 22]. Endoscopists' experience as a risk factor is controversial, as some prospective studies have reported it as a significant factor $[23,24]$, while others have not $[4,25]$. However, in a recent meta-analysis, high-volume endoscopists had $31 \%$ lower odds for complications than low-volume endoscopists [26]. In our study, the experience of the endoscopists as a confounding factor was minimal, as only experienced endoscopists performed ERCPs and trainees were involved in only a minority of procedures. The high overall success rate of $98.8 \%$ supports this argument.

The TPBS technique was first described by Goff in 1995 [27]. The advantage of TPBS over, for example needle-knife precut alone, is that the depth and location of the incision in relation to the CBD is more controlled [1]. The CBD lumen becomes visible in over half of the procedures [5]. In a prospective study by Kahaleh et al., the success rate with TPBS $(n=116)$ was $85 \%$ and, when combined with the needle-knife technique, this rose to $95 \%$ [28]. Furthermore, the overall complication rate in TPBS was $12 \%$ and the PEP rate $7.8 \%$, with no difference in PEP rate between conventional biliary sphincterotomy and TPBS.

The DGW method was first described by Dumonceau et al. in 1998 [29]. The technique has been used in cases of complex biliary cannulation, especially in patients with altered CBD anatomy due to neoplasia or atypical morphology of the ampulla $[10,29]$. A recent meta-analysis compared DGW with other techniques. No significant difference was found in CBD cannulation success; however, TPBS was not included as a comparator technique. DGW significantly increased the risk of PEP [13]. In a Japanese study, the PEP rate was $22 \%$ with DGW alone and $4.7 \%$ when combined with prophylactic pancreatic stenting. No pancreatic sphincterotomy was performed [11]. A very low PEP rate ( $2 \%)$ was reported in a prospective study of 50 patients undergoing DGW in Finland, even though none of the procedures included prophylactic pancreatic stenting [30].

The ESGE guideline recommends prophylactic pancreatic stenting when the guidewire inadvertently enters the PD [22]. In our study, only a minority of patients received a prophylactic pancreatic stent ( Table 1 ). This was due to the study protocol, which allowed the endoscopist to decide whether or not to place a stent. Even though prophylactic stents were seldom used, the overall PEP rate was comparable between the two techniques. In two recent studies, prophylactic pancreatic stenting did not affect the PEP rate $[31,32]$.
In a South Korean randomized study, TPBS had a lower PEP rate than DGW (10.8\% vs. 38.2\%) [8]. Sugiyama et al. compared TPBS and DGW, both with prophylactic pancreatic stents, in a randomized controlled trial of 34 patients per group [16]. The cannulation success rate was significantly higher with TPBS (94.1\%) than with DGW (58.8\%), but the PEP rate was the same in both groups, at $2.9 \%$. In the present study, when the randomized method failed, TPBS was used as a rescue method after DGW in $90.0 \%$ of cases, of which $77.8 \%$ were successful. In the TPBS group, DGW was used as a rescue method in 62.5 $\%$ of the patients and success was achieved in half of the cases. Therefore, both are feasible rescue methods when the guidewire is inserted into the PD.

The appearance and size of the papilla, and presence of a periampullary diverticulum or tumor infiltration may influence the outcome of biliary cannulation. In difficult cannulation cases with presence of a periampullary diverticulum, ESGE recommends selection of cannulation method according to the experience of the endoscopist and the anatomy of the patient [1]. It is difficult or sometimes impossible to proceed with TPBS in patients with intradiverticular papilla or a papilla located in the margin of the diverticulum because of the increased risk of bowel perforation. We suggest that the DGW technique is probably preferable in these situations; however, no definitive data supporting these preferences have been reported.

PD stent placement or use of the DGW technique may secure the papilla in an accessible position. Small or protruding papillae were more difficult to cannulate in a study of 1401 native papillae [33]. ESGE suggests considering the TPBS technique for small papillae in difficult cannulation. However, we prefer DGW for small papillae because of the potential increased risk of perforation with a precutting technique. Prospective comparative studies are warranted to determine the best methods of cannulating papillae with different appearances and in the presence of diverticulum.

Concerns related to long-term complications of TPBS have been expressed $[22,34]$. A recent retrospective case-control study from Helsinki found similar long-term complication rates between the TPBS group and a control group in which only biliary sphincterotomy was performed [35]. We plan to perform a follow-up study of the present multicenter trial in order to assess the long-term adverse events associated with TPBS. Our present results suggest a preference for TPBS as an advanced method in difficult cannulation; however, the power calculation was not performed to assess the cannulation success rate. Further prospective studies are warranted to seek the preferable cannulation method in difficult biliary cannulation.

\section{Limitations of the study}

Although our study is the largest of its kind, consisting of 203 patients, post hoc analysis (see supplementary material) revealed that when comparing the real crossover rates and PEP rates used in sample size calculation, our result is still slightly statistically underpowered $(67 \%-72 \%)$ to correctly reject the null hypothesis at the type I error rate.

The ESGE guideline on PEP prophylaxis with rectal NSAIDs was included in our study protocol as a recommendation. Pan- 
creatic stents were used at the discretion of the endoscopists. Only $78.8 \%$ of patients in the TPBS group and $82.8 \%$ in the DGW group received rectal NSAIDs prior to ERCP. In addition, prophylactic pancreatic stents were seldom used. This might increase the risk of PEP, although there was no difference in prophylaxis between the two study groups. Neither was there a difference in the PEP rate in randomized patients between those who received rectal NSAIDs and those who did not.

It was not possible to randomize all the patients fulfilling the criteria of difficult cannulation. If the guidewire did not enter the PD, patients were not randomized or analyzed as difficult procedures. Median cannulation times between the two study groups were comparable but it should be noted that maximum cannulation times were more than 1 hour in both groups with repetition in the randomized method. Outside clinical trials, these patients would undergo additional cannulation methods sooner to gain access to the CBD. Successful cannulation following crossover between the randomized methods was not considered as a success in the study analysis.

The number of patients recruited and randomized varied between centers, resulting in a potential selection bias in the results.

The protocol time limit of 15 minutes for cannulation attempts using the randomized method was not followed in 16 procedures. It seems that in clinical practice this time limit was too long to follow when the method appeared unsuccessful.

According to the study protocol, ERCP had to be performed by an expert. Only centers that were familiar with both techniques participated in the study. However, in 4/104 TPBS procedures and 2/99 DGW procedures, trainees were involved, and this may prolong the cannulation time unnecessarily. The definition of difficult cannulation was based on a study in which only experts performed ERCP [3]; thus, the time limit of 5 minutes may be too strict in regular clinical practice involving trainees.

A 30-day follow-up was carried out to verify patient records. Minor adverse events may have been missed if a patient did not contact the hospital after discharge.

\section{Conclusion}

TPBS and DGW resulted in similar PEP rates. The cannulation success rate with TPBS seemed to be higher than that with DGW. Prospective comparative studies are warranted to determine the best method for cannulation of papillae with different appearances and in the presence of diverticulum. TPBS is a good alternative in cases of difficult cannulation when the guidewire is in the PD; however, it remains a method for experienced endoscopists only.

\section{Acknowledgments}

Vilja Koskensalo would like to thank The Finnish Medical Foundation (1862/2018) and The Maud Kuistila Memorial Foundation (2019-0366F) for the working grants. The authors would like to thank statistician Pasi Aronen from the Biostatistical
Consulting Unit, Helsinki University Hospital and University of Helsinki, for his valuable contribution to the statistical analyses.

\section{Funding}

Maud Kuistilan Muistosäätiö

2019-0366F

Suomen Lääketieteen Säätiö

$1862 / 2018$

Clinical trial

https://clinicaltrials.gov | Registration number (trial ID): NCT02548884 | Type of study: Randomized, multicenter study

\section{Competing interests}

The authors declare that they have no conflicts of interests.

References

[1] Testoni PA, Mariani A, Aabakken L et al. Papillary cannulation and sphincterotomy techniques at ERCP: European Society of Gastrointestinal Endoscopy (ESGE) Clinical Guideline. Endoscopy 2016; 48: 657-683

[2] Dumonceau J-M, Andriulli A, Elmunzer B] et al. Prophylaxis of postERCP pancreatitis: European Society of Gastrointestinal Endoscopy (ESGE) Guideline - updated June 2014. Endoscopy 2014; 46: 799-815

[3] Halttunen J, Meisner S, Aabakken L et al. Difficult cannulation as defined by a prospective study of the Scandinavian Association for Digestive Endoscopy (SADE) in 907 ERCPs. Scand J Gastroenterol 2014; 49: 752-758

[4] Testoni PA, Mariani A, Giussani A et al. Risk factors for post-ERCP pancreatitis in high- and low-volume centers and among expert and non-expert operators: a prospective multicenter study. Am J Gastroenterol 2010; 105: 1753-1761

[5] Halttunen J, Keranen I, Udd M et al. Pancreatic sphincterotomy versus needle knife precut in difficult biliary cannulation. Surg Endosc 2009; 23: $745-749$

[6] Cotton PB, Lehman G, Vennes J et al. Endoscopic sphincterotomy complications and their management: an attempt at consensus. Gastrointest Endosc 1991; 37: 383-393

[7] Goff JS. Long-term experience with the transpancreatic sphincter precut approach to biliary sphincterotomy. Gastrointest Endosc 1999; 50: 642-645

[8] Yoo YW, Cha SW, Lee WC et al. Double guidewire technique vs transpancreatic precut sphincterotomy in difficult biliary cannulation. World J Gastroenterol 2013; 19: 108-114

[9] Angsuwatcharakon P, Rerknimitr R, Ridtitid W et al. Success rate and cannulation time between precut sphincterotomy and double-guidewire technique in truly difficult biliary cannulation. J Gastroenterol Hepatol 2012; 27: 356-361

[10] Herreros de Tejada A, Calleja JL, Diaz G et al. Double-guidewire technique for difficult bile duct cannulation: a multicenter randomized, controlled trial. Gastrointest Endosc 2009; 70: 700-709

[11] Ito K, Fujita N, Noda Y et al. Pancreatic guidewire placement for achieving selective biliary cannulation during endoscopic retrograde 
cholangio-pancreatography. World J Gastroenterol 2008; 14: 55955600

[12] Hennekens CH, Buring SE, Mayrent SL. Epidemiology in medicine. Boston, USA: Little, Brown \& Co; 1987

[13] Tse F, Yuan Y, Moayyedi P et al. Double-guidewire technique in difficult biliary cannulation for the prevention of post-ERCP pancreatitis: a systematic review and meta-analysis. Endoscopy 2017; 49: 15-26

[14] Catalano MF, Linder JD, Geenen JE. Endoscopic transpancreatic papillary septotomy for inaccessible obstructed bile ducts: comparison with standard pre-cut papillotomy. Gastrointest Endosc 2004; 60: 557-561

[15] Lee YJ, Park YK, Lee MJ et al. Different strategies for transpancreatic septotomy and needle knife infundibulotomy due to the presence of unintended pancreatic cannulation in difficult biliary cannulation. Gut Liver 2015; 9: 534-539

[16] Sugiyama H, Tsuyuguchi T, Sakai Y et al. Transpancreatic precut papillotomy versus double-guidewire technique in difficult biliary cannulation: prospective randomized study. Endoscopy 2018; 50: 33-39

[17] Zang J, Zhang C, Gao J. Guidewire-assisted transpancreatic sphincterotomy for difficult biliary cannulation: a prospective randomized controlled trial. Surg Laparosc Endosc Percutan Tech 2014; 24: 429433

[18] Freeman ML, Guda NM. Prevention of post-ERCP pancreatitis: a comprehensive review. Gastrointest Endosc 2004; 59: 845-864

[19] Andriulli A, Loperfido S, Napolitano G et al. Incidence rates of postERCP complications: a systematic survey of prospective studies. Am J Gastroenterol 2007; 102: 1781-1788

[20] Kapral C, Mühlberger A, Wewalka F et al. Quality assessment of endoscopic retrograde cholangiopancreatography. Eur J Gastroenterol Hepatol 2012; 24: 1447-1454

[21] Parekh PJ, Majithia R, Sikka SK et al. The "Scope" of post-ERCP pancreatitis. Mayo Clin Proc 2017; 92: 434-448

[22] Dumonceau JM, Kapral C, Aabakken L et al. ERCP-related adverse events: European Society of Gastrointestinal Endoscopy (ESGE) Guideline. Endoscopy 2020; 52: 127-149

[23] Cheng CL, Sherman S, Watkins JL et al. Risk factors for post-ERCP pancreatitis: a prospective multicenter study. Am J Gastroenterol 2006; 101: 139-147

[24] Lee H, Cho CM, Heo J et al. Impact of hospital volume and the experience of the endoscopist on adverse events related to endoscopic retrograde cholangiopancreatography: a prospective observational study. Gut Liver 2020; 14: 257-264

[25] Freeman ML, DiSario JA, Nelson DB et al. Risk factors for post-ERCP pancreatitis: a prospective, multicenter study. Gastrointest Endosc 2001; 54: 425-434

[26] Keswani RN, Qumseya B], O’Dwyer LC et al. Association between endoscopist and center endoscopic retrograde cholangiopancreatography volume with procedure success and adverse outcomes: a systematic review and meta-analysis. Clin Gastroenterol Hepatol 2017; 15: $1866-1875$

[27] Goff JS. Common bile duct pre-cut sphincterotomy: transpancreatic sphincter approach. Gastrointest Endosc 1995; 41: 502-505

[28] Kahaleh M, Tokar J, Mullick T et al. Prospective evaluation of pancreatic sphincterotomy as a precut technique for biliary cannulation. Clin Gastroenterol Hepatol 2004; 2: 971-977

[29] Dumonceau JM, Deviere J, Cremer M. A new method of achieving deep cannulation of the common bile duct during endoscopic retrograde cholangiopancreatography. Endoscopy 1998; 30: S80

[30] Gronroos JM, Vihervaara H, Gullichsen R et al. Double-guidewire-assisted biliary cannulation: experiences from a single tertiary referral center. Surg Endosc 2011; 25: 1599-1602

[31] Lee YS, Cho CM, Cho KB et al. Difficult biliary cannulation from the perspective of post-endoscopic retrograde cholangiopancreatography pancreatitis: identifying the optimal timing for the rescue cannulation technique. Gut Liver 2020: doi:10.5009/gnl19304

[32] Sotoudehmanesh R, Ali-Asgari A, Khatibian M et al. Pharmacological prophylaxis versus pancreatic duct stenting plus pharmacological prophylaxis for prevention of post-ERCP pancreatitis in high risk patients: a randomized trial. Endoscopy 2019; 51: 915-921

[33] Haraldsson E, Kylanpaa L, Gronroos J et al. Macroscopic appearance of the major duodenal papilla influences bile duct cannulation: a prospective multicenter study by the Scandinavian Association for Digestive Endoscopy Study Group for ERCP. Gastrointest Endosc 2019; 90: 957-963

[34] Pécsi D, Farkas N, Hegyi P et al. Transpancreatic sphincterotomy is effective and safe in expert hands on the short term. Dig Dis Sci 2019; 64: $2429-2444$

[35] Koskensalo V, Udd M, Rainio M et al. Transpancreatic biliary sphincterotomy for biliary access is safe also on a long-term scale. Surg Endosc 2020: doi:10.1007/s00464-019-07364-y 